\title{
Hard X-ray polarimetry with Astrosat-CZTI
}

\author{
S. V. Vadawale ${ }^{1}$, T. Chattopadhyay ${ }^{1,2}$, A. R. Rao ${ }^{3}$, D. Bhattacharya ${ }^{4}$, V. B. Bhalerao ${ }^{4}$, N. Vagshette $^{4}$, \\ P. Pawar ${ }^{5}$, and S. Sreekumar ${ }^{6}$
}

\author{
1 Physical Research Laboratory, Navarangpura, 380009 Ahmedabad, India \\ e-mail: santoshv@prl.res.in \\ 2 Indian Institute of Technology Gandhinagar, 382424 Ahmedabad, India \\ 3 Tata Institute of Fundamental Research, Homi Bhabha Road, Colaba, 400005 Mumbai, India \\ ${ }^{4}$ Inter-University Centre for Astronomy and Astrophysics, Post Bag 4, 411007 Pune, India \\ 5 Swami Ramanand Teerth Marathwada University, 431604 Nanded, India \\ 6 Vikram Sarabhai Space Centre, VRC, 695022 Thiruvananthapuram, India
}

Received 19 January 2015 / Accepted 9 April 2015

\section{ABSTRACT}

\begin{abstract}
X-ray polarimetry is largely an unexplored area of an otherwise mature field of X-ray astronomy. Except for a few early attempts during the 1970s, no dedicated X-ray polarimeter has been flown during the past four decades. On the other hand, the scientific value of X-ray polarization measurement has been well known for a long time, and there has been significant technical progress in developing sensitive X-ray polarimeters in recent years. But there are no approved dedicated X-ray polarimetric experiments to be flown in the near future, so it is important to explore the polarimetric capabilities of other existing or planned instruments and examine whether they can provide significant astrophysical polarization measurements. In this paper, we present experimental results to show that the CZTI instrument onboard the forthcoming Indian astronomy mission, Astrosat, will be able to provide sensitive measurements of X-ray polarization in the energy range of 100-300 keV. CZTI will be able to constrain any intrinsic polarization greater than $\sim 40 \%$ for bright X-ray sources ( $>500 \mathrm{mCrab}$ ) within a short exposure of $\sim 100 \mathrm{ks}$ with a 3 -sigma confidence level. We show that this seemingly "modest" sensitivity can play a very significant role in addressing long pending questions, such as the contribution of relativistic jets to hard X-rays in black hole binaries and X-ray emission mechanism and geometry in X-ray pulsars.
\end{abstract}

Key words. instrumentation: polarimeters $-\mathrm{X}$-rays: individuals: Cygnus X-1 - X-rays: individuals: Crab - techniques: photometric

\section{Introduction}

Polarization is a very important property of radiation from astrophysical sources, and it carries unique information regarding the emission mechanism, physical conditions, and emission geometry at the source. The importance of polarization measurement was realized in the very early days of X-ray astronomy (Novick 1975; Meszaros et al. 1988; Kallman 2004; Krawczynski et al. 2011, and references therein), and there is a large body of theoretically expected polarization signatures from different types of X-ray sources.

Despite the tremendous progress in other fields of X-ray astronomy, however, the field of X-ray polarimetry has been largely unexplored observationally (Weisskopf 2010). The primary reason for this is the extremely photon-hungry nature of polarimetry (Weisskopf et al. 2010; Elsner et al. 2012), which along with the low efficiency and other technical difficulties of different polarization measurement methods, result in low sensitivity for a dedicated polarimetric mission compared to the traditional spectroscopic, timing, or imaging missions of comparable size and cost. Consequently, there is no approved mission dedicated to X-ray polarimetry, despite many proposed missions and balloon-borne experiments (McConnell et al. 2009; Costa et al. 2010; Tagliaferri et al. 2010; Pearce et al. 2012; Soffitta et al. 2013; Guo et al. 2013; Kislat et al. 2013; Dong 2014), even though some of these missions have reached an advanced stage in the selection process (Rishin et al. 2010). On the other hand, there is growing realization in recent times that thanks to the improved techniques of polarization, a dedicated polarimetry mission would provide very fresh results. A dedicated X-ray polarimetry mission GEMS (Jahoda 2010) was selected for development as part of the NASA Small Explorer program in 2009, but was discontinued in 2012. It was proposed again at the 2014 SMEX NASA call, which also attracted two more proposals dedicated to X-ray polarimetry, indicating enhanced interest of community in firm X-ray polarization measurements. Still, in the absence of any approved dedicated mission, it is very important to explore any possibility of obtaining meaningful X-ray polarization measurements from instruments designed for other related purposes (such as X-ray timing and spectroscopy) and to have a robust ground calibration.

There have been many attempts to retrieve polarimetric information from the existing X-ray instruments that have notional polarimetric sensitivity, and the notable results are measurement of highly polarized emission from a gamma-ray burst (GRB) with the RHESSI mission and from two Galactic X-ray sources (Cygnus X-1, a black hole binary, and Crab, a pulsar wind nebula) with the IBIS and the SPI instruments onboard the Integral mission (Coburn \& Boggs 2003; Forot et al. 2008; Laurent et al. 2011; Jourdain et al. 2012). These measurements, however, faced some criticism because these instruments are not fully optimized for polarimetry and particularly because their polarimetric capabilities were not calibrated before launch (Rutledge \& Fox 2004; Zdziarski et al. 2014).

Here we show by experiment that the Cadmium-ZincTelluride Imager (CZTI) onboard the forthcoming Indian astronomy mission Astrosat will be capable of measuring polarization 


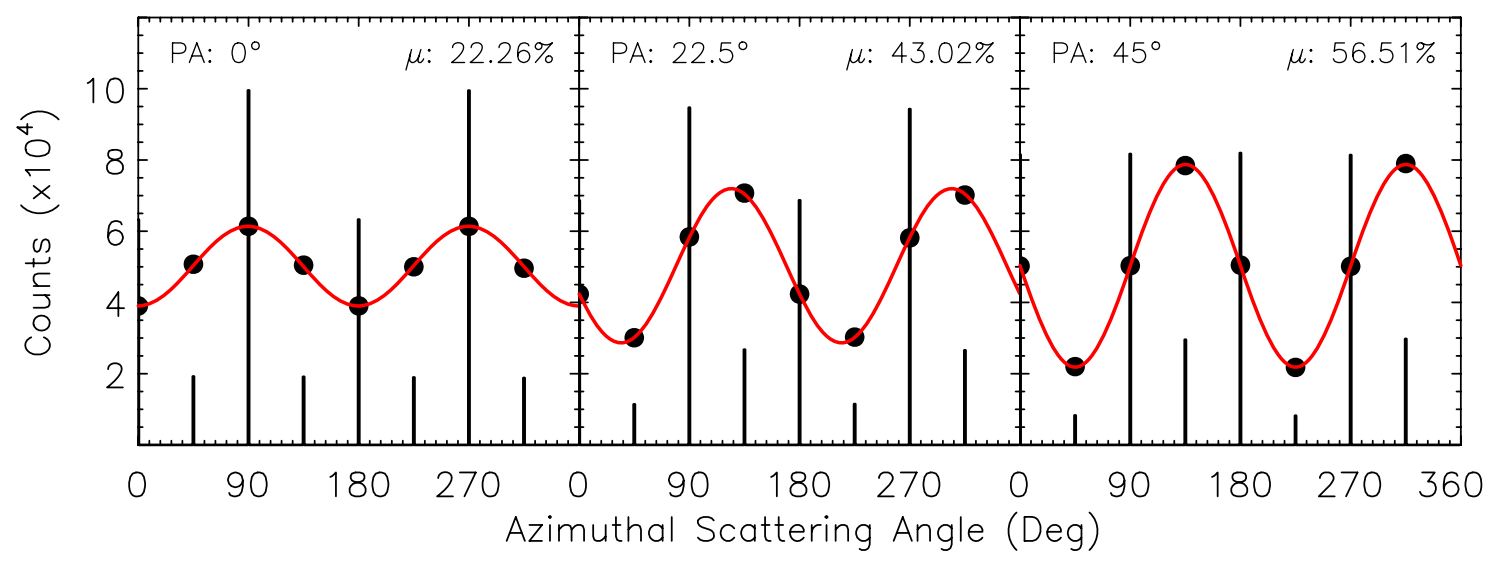

Fig. 1. Simulated histograms of azimuthal scattering angles for three polarization angles $0^{\circ}, 22.5^{\circ}$, and $45^{\circ}$ with reference to the pixel edge for a 100\% polarized beam. The vertical lines show uncorrected histograms that show the effect of unequal angular bins. Black dots show corrected values for each bin (see Eq. (1)), and the red lines represents fits (Eq. (2)) to these points.

of the incident $\mathrm{X}$-rays in the energy range $100-300 \mathrm{keV}$. In the next section, we discuss the basic concepts of X-ray polarimetry with CZTI, which is followed by discussion of the experiment and the results that demonstrate the polarimetric capability of CZTI. Following this, we discuss the overall polarimetric sensitivity of CZTI with a realistic polarimetric background in Sect. 4 and the astrophysical significance of the polarimetric capability of CZTI in Sect. 5, before concluding in Sect. 6.

\section{X-ray polarimetry with Astrosat-CZTI}

Astrosat is an Indian multiwavelength astronomy mission scheduled to be launched in late 2015. It consists of five instruments that cover a range of spectrum from optical to hard X-rays (Singh et al. 2014; Agrawal 2006). The Cadmium-ZincTelluride Imager (CZTI) onboard Astrosat, which is primarily designed for hard X-ray imaging and spectroscopy, is one instrument expected to be sensitive to the polarization of the incident X-rays thanks to its large pixilated detector plane. The CZTI is a coded aperture mask telescope with a total active area of $1024 \mathrm{~cm}^{2}$. The detector plane consists of 64 detector modules arranged in four identical quadrants, each having 16 modules arranged in a square geometry. Each module is an independent cadmium-zinc-telluride $(\mathrm{CZT})$ detector with a $16 \times 16$ array of pixels, each $2.5 \mathrm{~mm} \times 2.5 \mathrm{~mm}$. The $5 \mathrm{~mm}$ detector thickness provides detection efficiency at energies higher than the primary spectroscopic energy range of $10 \mathrm{keV}$ to $100 \mathrm{keV}$, where a significant fraction of photon interaction occurs by means of Compton scattering. In these events, the Compton-scattered photon could be detected in an adjacent pixel. Since the direction of the scattered photon depends on the polarization direction of the incident photon, such pixelated detectors can, in general, and for Astrosat-CZTI, in particular, be used as a Compton X-ray polarimeter (Chattopadhyay et al. 2014, hereafter C14). In the energy range of interest for CZTI, 100-300 keV, the energy of the recoil electron is much lower than the energy of the scattered photon. It is therefore assumed that the pixel with the lower energy deposition is the scattering pixel and that the second pixel is the absorbing pixel.

Another important criterion determined by $\mathrm{C} 14$ for selecting the valid double pixel events is to require that the ratio of energies deposited in two adjacent pixels be greater than 2 . The azimuthal angle of the Compton scattering is determined from the direction of the center of the scattering pixel to the center of the absorbing pixel with reference to a predefined instrument reference plane. The histogram of the azimuthal scattering angles over eight angular bins, corresponding to the four edge pixels, and the four corner pixels can be used to quantify the scattering modulation. It should be noted that this histogram has an inherent modulation owing to unequal angular bins that correspond to the edge and the corner pixels. However, this inherent modulation pattern can be corrected by normalizing the measured azimuthal distribution with the azimuthal distribution for unpolarized beam (Lei et al. 1997; Krawczynski et al. 2011).

If $N_{i, \text { pol }}$ is the number of counts in the $i$ th bin for $100 \%$ polarized beam, $M_{i \text {,unpol }}$ is the counts in that bin for $100 \%$ unpolarized beam, and $\bar{M}_{\text {unpol }}$ is mean counts, then normalized counts in the $i$ th bin are given by

$N_{i, \mathrm{cor}}=\frac{N_{i, \mathrm{pol}}}{M_{i, \mathrm{unpol}}} \bar{M}_{\mathrm{unpol}}$.

The corrected azimuthal modulation pattern, the so-called modulation curve, can then be fit by

$C(\phi)=A \cos ^{2}\left(\phi-\phi_{0}\right)+B$,

the modulation amplitude is then defined by

$\mu=\frac{A}{A+2 B}$.

Figure 1 shows the corrected and uncorrected modulation curves for three different polarization angles with reference to the pixel edge. It can be seen that the modulation factors, obtained by fitting Eq. (2) to the corrected modulation curves, are different for different polarization angles. This results in different polarization sensitivities at different polarization angles, as discussed in C14 (also see Sect. 4).

The sensitivity of an X-ray polarimeter is specified in terms of minimum detectable polarization (MDP) given by

$M D P_{99 \%}=\frac{4.29}{\mu_{100} R_{\mathrm{src}}} \sqrt{\frac{R_{\mathrm{src}}+R_{\mathrm{bkg}}}{T}}$,

where $R_{\mathrm{src}}$ and $R_{\mathrm{bkg}}$ are the source and background count rates, $T$ is the exposure time, and $\mu_{100}$, the so-called the modulation factor, is the modulation amplitude for a $100 \%$ polarized beam.

Based on extensive simulations, we had shown in $\mathrm{C} 14$ that the Astrosat-CZTI will have significant polarization measurement capabilities in the energy range of $100-300 \mathrm{keV}$, particulalry for bright (>500 mCrab) X-ray sources. 

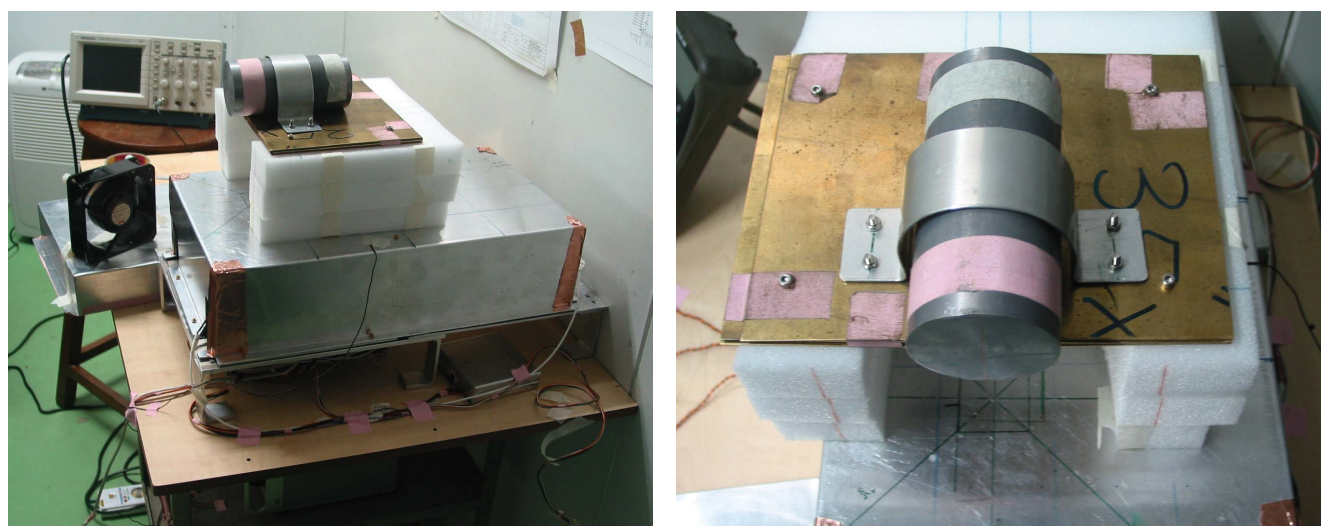

Fig. 2. Polarization experiment setup with CZTI (left) and zoomed view of the setup (right).

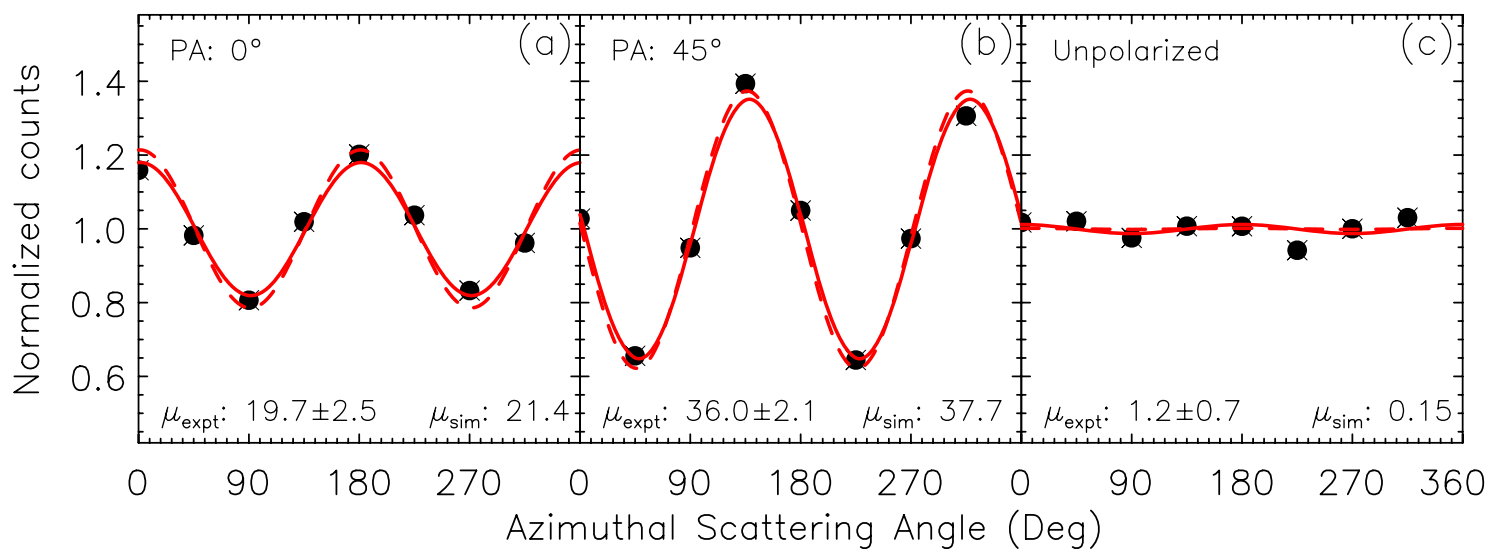

Fig. 3. a) and b) Experimentally measured modulation curves for partially polarized X-rays from ${ }^{133}$ Ba source at two polarization angles, $0^{\circ}$ and $45^{\circ}$ respectively. c) The same curve measured for unpolarized X-rays from ${ }^{133} \mathrm{Ba}$. The dashed line shows the modulation curves obtained from the Geant 4 simulations of the experimental setup.

\section{Experimental confirmation}

To confirm the polarimetric capability of CZTI, we carried out an X-ray polarization measurement experiment using the actual flight model of CZTI module, which ensures that all properties of flight electronics are also included in the experiment. We produced a partially polarized beam of X-rays by scattering $356 \mathrm{keV}$ $\mathrm{X}$-rays from the radioactive source ${ }^{133} \mathrm{Ba}$ at $\sim 90$ degrees (see Fig. 2). A $6 \mathrm{~cm}$-long aluminum cylinder was used as a scatterer. The source and the scatterer were placed inside a $4 \mathrm{~cm}$ thick lead cylinder with a slit of $5 \mathrm{~cm}$ length and $2 \mathrm{~mm}$ width. The CZTI module is kept below the slit such that only the photons from ${ }^{133} \mathrm{Ba}$ scattered at $90^{\circ}$ by the aluminum scatterer can reach the CZTI detector. With this setup, the angle of scattering is constrained to $90^{\circ} \pm 15^{\circ}$ implying a partially polarized beam of energy between $190 \mathrm{keV}$ and $240 \mathrm{keV}$.

The basic data filtering and analysis were carried out as discussed in C14. This involved selection of appropriate adjacent double-pixel events, generating eight-bin azimuthal distribution with reference to lower energy pixels and correcting azimuthal distribution for the unequal angle bins. Figure 3 shows the corrected modulation curves for partially polarized beam at two different polarization angles, $0^{\circ}$ and $45^{\circ}$, with reference to one of the edges of the CZT detector module, as well as for an unpolarized beam (direct exposure to $356 \mathrm{keV} \mathrm{X}$-rays).

It can be seen that the modulation curves for the measurements at these polarization angles show a clear signature of the polarization of the incident beam and the modulation curves corresponding to different polarization angles also change, as expected. The errors in the observed modulations are nominal $1 \sigma$ errors and the simulation results too have errors of similar magnitudes. In the case of the unpolarized beam, the residual modulation, which could be instrumental artifacts, is almost neglible (1\%), and thus will not have any significant effect on the polarization measurement aimed at a largely polarized source. It should be noted here that one major criticism of the recent X-ray polarization measurements by INTEGRAL IBIS and SPI instruments is that these instruments were never tested with an unpolarized beam.

These results clearly demonstrate that CZTI is capable of detecting polarization of the incident X-rays. However, the observed modulation amplitude cannot be directly used to validate the simulation because of the partially polarized beam. To have a quantitative comparison with the simulations, we repeated the simulations for the exact geometry of the experiment including the generation of the polarized beam and considered the experimentally measured background to calculate the modulation factor. The modulation factors obtained from these simulations typically agree within $\sim 1 \%$ of the experimentally measured modulation factors in all cases. This implies that the value of $\mu_{100}$ obtained from simulations do represent a realistic modulation factor.

\section{Polarimetric background and sensitivity}

The confidence provided by the experimental verification of the polarization measurement capability of CZTI enables us to use 

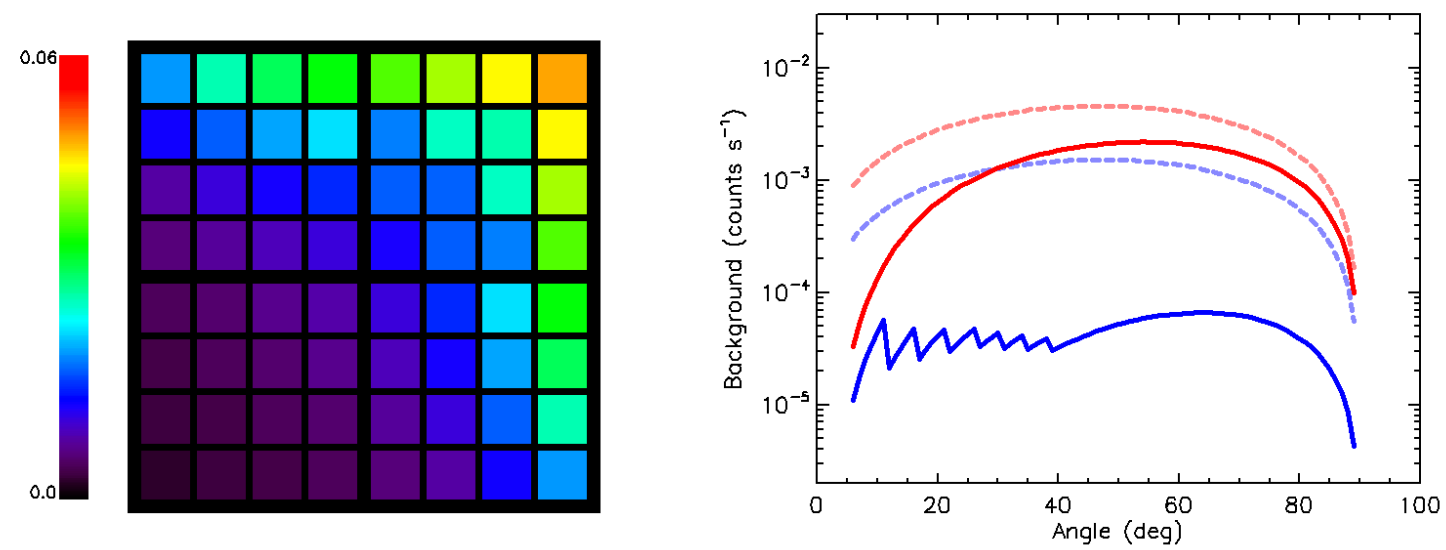

Fig. 4. Polarimetric background in the energy range of 100-300 keV expected for individual detector modules of CZTI (left), and angle-dependent contribution of diffuse X-ray background to the total background for the innermost (blue) and outermost module (red) to demonstrate the effect of collimators and module location (right). The dashed curves show background without considering module-wise collimators.
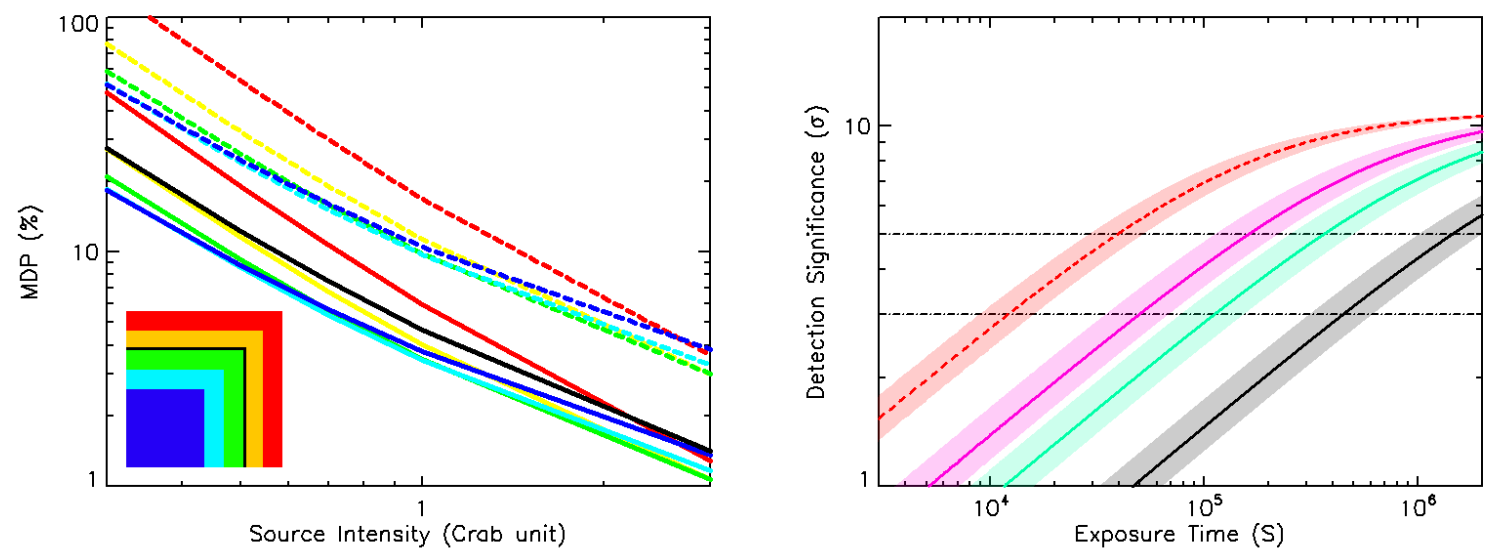

Fig. 5. Polarimetric sensitivity of CZTI in terms of MDP (left) as a function of source intensity for exposure time of 1 Ms. The dashed and solid lines represent the polarization angle aligned with the pixel edges and at $45^{\circ}$, respectively with the latter more sensitive owing to a higher modulation factor, as expected. Different colors represent subsamples of CZTI detector plane as shown in the inset. The black line represents the overall MDP as mentioned in the text corresponding to average $22.5^{\circ}$ polarization and the third (green) subarray. Right: detection significance of polarization measurement for highly polarized bright sources. The bottom three lines correspond to $500 \mathrm{mCrab}$ sources with polarization fraction of $20 \%$ (black), $40 \%$ (green), and 60\% (pink), respectively. The top line (dashed red) corresponds to one Crab source that has a polarization fraction of $50 \%$.

the simulation methods to quantify the actual polarimetric sensitivity of CZTI in the astrophysical context. In the case of CZTI, the sensitivity is determined by the polarimetric background, $R_{\mathrm{bkg}}$, because the background is primarily dominated by the Compton scattering of the diffuse X-ray background as shown in $\mathrm{C} 14$ and can be as high as the source count rate. This is because the primary spectroscopic energy range for the CZTI is up to $100 \mathrm{keV}$, whereas the polarization measurements are possible only at energies $>100 \mathrm{keV}$ because of the requirement to detect the primary scattering event. The coded mask, as well as the shielding and other support structures, therefore gets increasingly transparent in the polarimetric energy range of CZTI. The high polarimetric background also results in degradation of the modulation curve. Therefore, to determine the unknown degree of polarization of the incident X-rays, the actual modulation factor, $\mu_{100}$, has to be replaced by a degraded modulation factor, $\mu_{\mathrm{d}, 100}$. Both these aspects were considered in detail in $\mathrm{C} 14$, and it was found that CZTI will have significant polarimetric capabilities despite having semi-transparent shielding. The total polarimetric background (i.e., total double pixel events passing all the filtering criteria) was almost the same as the total polarimetric count rate from Crab. It was pointed out to us (Costa, priv. comm.) that calculations of $\mathrm{C} 14$ did not include background owing to the Earth's X-ray albedo, which is now included in our revised calculations. We assume that the albedo background is not polarized, which is a fair assumption because of the low Earth orbit of Astrosat and the resulting azimuthal symmetry in scattering of albedo from the Earth's atmosphere. Also we have significantly improved our calculations to include effective shielding at an individual detector module level. This enables us to calculate the module-wise polarimetric background as shown in Fig. 4 and thus provide a more reliable estimate of effective background.

The estimated effective background is now $\sim 1.5 \mathrm{Crab}$, so the expected polarimetric sensitivity, as shown in Fig. 5 in terms of MDP as a function of source intensity, is slightly less than presented in C14. One important repercussion of our module-wise background calculation is the realization that different subsets of the CZTI detector plane will have different sensitivities according to the net background for the given subset. This provides 
a very powerful handle to cross-verify the actual polarization measurement between different subsets and thus to have enhanced confidence in the polarization measurement. It should be noted here that thanks to the availability of event wise data, all such subsampling, either in selecting module subset or the energy range, can be carried out at the analysis level and thus does not impose any special restriction on the actual observation.

Figure 5 shows the polarimetric sensitivity of CZTI in terms of MDP as a function of source intensity. It can be seen that for a Crab-like source, an MDP of 5\% can be achieved in an exposure time of $\sim 1 \mathrm{Ms}$ (million seconds). Though such long exposure times are routinely quoted in the context of X-ray polarimetry, these may not be realistic for the observatory class mission such as Astrosat. On the other hand, in the energy range of interest $(100-300 \mathrm{keV})$ the emission from almost all X-ray sources is expected to be of non-thermal origin and thus can be highly polarized. This is corroborated by the recent reports of more then $50 \%$ polarized soft gamma emission by Integral from two bright X-ray sources Crab and Cygnus X-1 (Forot et al. 2008; Laurent et al. 2011; Jourdain et al. 2012). In this context a more appropriate question is how well its polarization can be constrained by CZTI with a typical exposure time of a few tens of kiloseconds, given the highly polarized $\mathrm{X}$-ray emission,.

For an unknown source, the polarization degree $P$ is given by

$$
P=\frac{\mu}{\mu_{\mathrm{d}, 100}}
$$

where $\mu$ and $\mu_{\mathrm{d}, 100}$ are the measured modulation amplitude for that source and the degraded modulation factor respectively. It should be noted that the degraded modulation factor $\mu_{\mathrm{d}, 100}$ takes into account the effect of Compton scattering of the background photons (both cosmic X-ray background and albedo background) as discussed in $\mathrm{C} 14$, and as a result it is smaller than the $\mu_{100}$ obtained from Geant4 simulations (Agostinelli et al. 2003). Error in the measurement is given by

$\frac{\sigma_{P}}{P}=\sqrt{\frac{\sigma_{\mu}^{2}}{\mu^{2}}+\frac{\sigma_{\mu_{\mathrm{d}, 100}}^{2}}{\mu_{\mathrm{d}, 100^{2}}}}$,

where $\sigma_{\mu}$ is the statistical error associated with the measurement of modulation factor $\mu$, which depends on both the degree of polarization in the source and the source intensity. Here, $\sigma_{\mu_{\mathrm{d}, 100}}$ is the error in $\mu_{\mathrm{d}, 100}$.

It should be noted that $\sigma_{\mu_{\mathrm{d}, 100}}$ depends on the relative angle between the polarization direction of the incident X-rays and a reference direction of the instrument. In general, the polarization angle cannot be constrained to better than $28.5^{\circ} /\left(P / \sigma_{P}\right)$ simply by fitting the modulation curves (Strohmayer \& Kallman 2013). Thus in most cases, the uncertainty in polarization angle can be assumed to be $15^{\circ}-20^{\circ}$. Figure 5 shows the detection significance for different source intensities as a function of exposure time, with $\mu_{\mathrm{d}, 100}$ and $\sigma_{\mu_{\mathrm{d}, 100}}$ corresponding to the average polarization angle of $22.5^{\circ}$ and uncertainty in polarization angle of $\pm 10^{\circ}$. It can be seen that for long exposure times, the significance of polarization measurement saturates due to the uncertainty in $\mu_{\mathrm{d}, 100}$. However, for shorter exposure times (100 ks), the uncertainty in polarization measurement is dominated by the counting statistics.

Since the long exposure times are expected to be the result of multiple shorter exposure times with the different position angles of the spacecraft, it is more appropriate to consider the average polarization angle of $22.5^{\circ}$ (the angle with reference to the instrument reference direction and not the absolute polarization angle in the sky frame). However, in many such cases, it might be possible to have tighter constraints on the absolute polarization angle based on multiple smaller subsets of the same data, which may result in higher significance for the polarization measurement.

Another factor affecting detection significance of polarization is the uncertainty in polarimetric background. The shaded regions around the lines in Fig. 5 represent the variation in significance for the probable variation in the polarimetric background by $\pm 20 \%$ for the same statistical and systematic errors. It can be seen that, even after considering all these uncertainties, the polarization can be measured with $3 \sigma$ confidence with an exposure time of $100-150 \mathrm{ks}$ for a $500 \mathrm{mCrab}$ source with a $40 \%$ intrinsic polarized fraction.

\section{Astrophysical significance of CZTI polarimetry}

Since polarization measurements with CZTI are possible only for bright $(>500 \mathrm{mCrab}) \mathrm{X}$-ray sources, the obvious candidates are the two eminent sources Cygnus X-1, a black hole binary, and Crab, a pulsar wind nebula. That the initial indication of highly polarized emission from both these sources is available from Integral observations makes the astrophysical significance of CZTI polarimetric capability more relevant.

For example, in the case of Cygnus X-1, measurements by both IBIS and SPI instruments on INTEGRAL show very high polarization fraction $(\sim 65 \%)$ at energies greater than $\sim 250 \mathrm{keV}$, which is interpreted in terms of the emission from the base of a jet (Laurent et al. 2011). While a significant contribution from the jet to hard X-ray/soft gamma emission has been speculated on for some other black hole binaries (Vadawale et al. 2001; Markoff et al. 2001; Vadawale et al. 2003), recent SED modelling of the Cygnus X-1 spectral energy distribution by Zdziarski et al. (2014), spanning the radio to $\mathrm{MeV}$ range, suggests that significant jet contribution to hard X-ray emission can be achieved only with rather unrealistic parameters.

On the other hand, there are other models that predict both state-independent high polarization (Romero et al. 2014) and moderate polarization only in the hard state (Schnittman \& Krolik 2010). Thus the present scenario of the polarized nature of hard X-ray emission from Cygnus X-1, both observationally and theoretically, is fairly confusing, so it is important to independently constrain it. In this context we considered various available predictions of X-ray polarization for Cygnus X-1 to investigate whether they can be delineated with CZTI, particularly in the presence of some of the systematic effects, such as uncertainty in background and uncertainty in the knowledge of polarization angle (see Fig. 6).

The possible scenarios considered here (denoted by red, green, black, and blue, respectively, in Fig. 6) are: a strong polarization signature ( $\sim 50 \%)$ in LHS (low hard state) due to relatively high jet contribution; comparatively low polarization fraction of $25 \%$ because of relatively lower jet contribution in LHS; $\sim 15 \%$ polarization in LHS that originated in the corona owing to inverse Compton scattering and high polarization (around 55\% in the high soft state) because of synchrotron radiation from the corona itself. We found that most of the scenarios can be constrained fairly accurately within an exposure time of $\sim 200 \mathrm{ks}$.

In the case of the Crab nebula, based on the polarization measurements at other wavelengths (including soft X-rays which is the only historic positive $\mathrm{X}$-ray polarization measurement from an astrophysical source, Weisskopf et al. 1978), it is well known that the nebular emission arises from synchrotron radiation. However, both the mechanism and the geometry of the emission from the pulsar itself are still not fully understood 

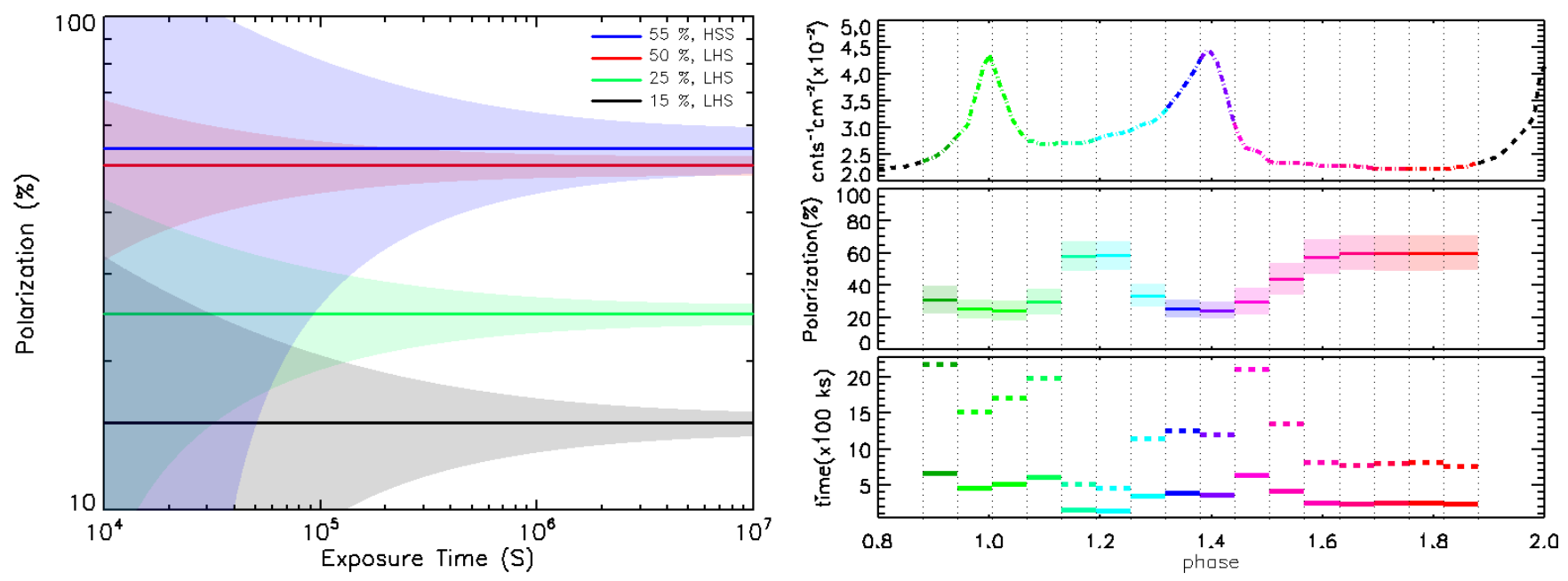

Fig. 6. Left: possible constraints on the hard X-ray polarization fraction of Cygnus X-1 for some of the model predictions proposed in literature (see text for details), predicting about $15 \%, 25 \%$, and $50 \%$ polarization in the low hard state (total flux $\sim 1$ Crab) and about $55 \%$ polarization in the high soft state (total flux $\sim 300 \mathrm{mCrab}$ ). Expected errors are shown as shaded regions. Right: feasibility of phase-resolved polarimetry with CZTI for Crab. The top panel shows Crab pulse profile in $100-300 \mathrm{keV}$. The middle panel shows polarization in 16 phases, as expected from an interpolation of the polarization measured by Integral in four regions of Crab pulse profile. The shaded region represents possible constraints on phase-wise polarization measurement with CZTI for a total exposure time of $1 \mathrm{Ms}$. The bottom panel shows the required exposure time to determine polarization for all phases with minimum 3 sigma (solid lines) and 5 sigma (dashed lines) confidence levels. It can be seen that with a total exposure time of $\sim 2 \mathrm{Ms}$, polarization in most of the phases can be constrained with better than 5-sigma confidence.

with various models, such as polar cap, outer gap, slot gap, and stripped wind (Dyks et al. 2004; Pétri \& Kirk 2005), which is compatible with all other observational characteristics. One distinguishing feature of these models is their phase-dependent polarization signature. The Integral measurements, folded with the Crab pulse period, suggest that the polarization varies at different phases and thus goes against the polar cap model. But to constrain other models, it is necessary to get truly phase-resolved polarization measurements, which typically need an order of magnitude longer exposure time. Since the Crab is likely to be observed for a long time as a standard candle, it is possible that a total exposure of a few million seconds can be obtained. In this case, we find that full phase-resolved polarization measurement is certainly possible with the lowest polarization also constrained better than 3 sigma. As an example, Fig. 6 shows the Crab polarization detection significance, estimated in 16 uniform phase bins. The phase-resolved polarization values used in our calculation are obtained by interpolating the recent Crab polarization estimates by IBIS (Moran et al. 2013). Thus we see that CZTI can provide much deeper insight into the emission mechanism of the Crab pulsar.

However, polarimetric studies with CZTI will not be limited to these two sources. There are many transient X-ray binaries, which undergo outbursts lasting from a few weeks to months and have intensities of more than $500 \mathrm{mCrab}$. Since such transient sources are of general interest, it is likely that at least the long outbursts will be observed extensively by Astrosat and thus will result in a total exposure time that is sufficient for the polarimetric studies with CZTI. Based on the analysis of long-term monitoring data from all sky monitors, such as RXTE-ASM, SWIFTBAT, and MAXI, we find that typically two to three outbursts that are brighter than $500 \mathrm{mCrab}$ and longer than one week are expected during the period of one year. Thus, given an operational life of five years, CZTI is likely to investigate the presence of highly polarized emission from at least ten transient sources that are typically black-hole and neutron-star binaries.

\section{Conclusion}

To summarize, Astrosat-CZTI will have significant polarimetric capabilities. The polarization capabilities are verified on the ground, both with partially polarized beam and more important, with an unpolarized beam. This will give additional confidence for considering the actual polarization measurements as intrinsic to the source and for constraining the various theoretical models based on them. The Gamma-ray Burst Polarimeter (GAP) instrument, which is a small Compton-scattering-based polarimeter onboard the Japanese Small Solar Power Sail Demonstrator mission IKAROS (Yonetoku et al. 2011), has demonstrated the importance of robust ground calibration of polarimeters, which is the basis for successfully measuring polarization of GRBs. The Soft Gamma Ray Detector (SGD), which is a narrowfield Compton telescope onboard the forthcoming Japanese mission Astro-H (Watanabe et al. 2012) to be launched next year, will also have a polarization measurement capability based on the Compton scattering principle in $80-300 \mathrm{keV}$ (Tajima et al. 2010). With its much larger area compared to GAP and much larger field of view compared to SGD, Astrosat-CZTI will prove to be a unique instrument capable of probing the X-ray polarization of bright celestial sources. Furthermore, since Astrosat is an observatory-class satellite with X-ray timing as one of its primary objectives, it is likely to devote a large fraction of its observing time to bright $\mathrm{X}$-ray binaries, thus facilitating measurement of X-ray polarisation in the $100-300 \mathrm{keV}$ region as an additional bonus. Thus it is likely that the field of hard X-ray polarimetry will witness significant advances in the near future.

\section{References}

Agostinelli, S., Allison, J., Amako, K., et al. 2003, Nucl. Instr. Meth. Phys. Res. A, 506, 250

Agrawal, P. C. 2006, Adv. Space Res., 38, 2989

Chattopadhyay, T., Vadawale, S. V., Rao, A. R., Sreekumar, S., \& Bhattacharya, D. 2014, Exp. Astron., 37, 555 
Coburn, W., \& Boggs, S. E. 2003, Nature, 423, 415

Costa, E., Bellazzini, R., Tagliaferri, G., et al. 2010, Exp. Astron., 28, 137

Dong, Y. 2014, SPIE Conf. Rev., 9144, 91443

Dyks, J., Harding, A. K., \& Rudak, B. 2004, ApJ, 606, 1125

Elsner, R. F., O’Dell, S. L., \& Weisskopf, M. C. 2012, Proc. SPIE, 8443, 84434

Forot, M., Laurent, P., Grenier, I. A., Gouiffès, C., \& Lebrun, F. 2008, ApJ, 688, L29

Guo, Q., Beilicke, M., Garson, A., et al. 2013, Astropart. Phys., 41, 63

Jahoda, K. 2010, SPIE Conf. Rev., 7732, 77320

Jourdain, E., Roques, J. P., Chauvin, M., \& Clark, D. J. 2012, ApJ, 761, 27

Kallman, T. 2004, Adv. Space Res., 34, 2673

Kislat, F., Beilicke, M., Baring, M. G., et al. 2013, IEEE Nucl. Sci. Symp./Med. Imag. Conf., 1

Krawczynski, H., Garson, A., Guo, Q., et al. 2011, Astropart. Phys., 34, 550

Laurent, P., Rodriguez, J., Wilms, J., et al. 2011, Science, 332, 438

Lei, F. R., Dean, A. J., \& Hills, G. L. 1997, Space Sci. Rev., 82, 309

Markoff, S., Falcke, H., \& Fender, R. 2001, A\&A, 372, L25

McConnell, M. L., Bancroft, C., Bloser, P. F., et al. 2009, SPIE Conf. Ser., 7435, 74350

Meszaros, P., Novick, R., Szentgyorgyi, A., Chanan, G. A., \& Weisskopf, M. C. 1988, ApJ, 324, 1056

Moran, P., Shearer, A., Gouiffes, C., \& Laurent, P. 2013, in An INTEGRAL view of the high-energy sky (the first 10 years) - 9th INTEGRAL Workshop, ArXiv e-print [arXiv: 1302 . 3622]

Novick, R. 1975, Space Sci. Rev., 18, 389

Pearce, M., Florén, H.-G., Jackson, M., et al. 2012, IEEE Nucl. Sci. Symp./ Med. Imag. Conf., 1885
Pétri, J., \& Kirk, J. G. 2005, ApJ, 627, L37

Rishin, P. V., Paul, B., Gopala Krishna, M. R., et al. 2010, In Proceedings of The First Year of MAXI: Monitoring Variable X-ray Sources, 371

Romero, G. E., Vieyro, F. L., \& Chaty, S. 2014, A\&A, 562, L7

Rutledge, R. E., \& Fox, D. B. 2004, MNRAS, 350, 1288

Schnittman, J. D., \& Krolik, J. H. 2010, ApJ, 712, 908

Singh, K. P., Tandon, S. N., Agrawal, P. C., et al. 2014, SPIE Conf. Ser., 9144, 91441

Soffitta, P., Barcons, X., Bellazzini, R., et al. 2013, Exp. Astron., 36, 523

Strohmayer, T., E., \& Kallman, T. R. 2013, ApJ, 773, 103

Tagliaferri, G., Argan, A., Bellazzini, R., et al. 2010, SPIE Conf. Ser., 7732, 773217

Tajima, H., et al. 2010, in X-ray Polarimetry: A New Window in Astrophysics (Cambridge University Press), 275

Vadawale, S. V., Rao, A. R., \& Chakrabarti, S. K. 2001, A\&A, 372, 793

Vadawale, S. V., Rao, A. R., Naik, S., et al. 2003, ApJ, 597, 1023

Watanabe, S., Tajima, H., Fukazawa, Y., et al. 2012, SPIE Conf. Ser., 8443 844326

Weisskopf, M. C. 2010, inn X-ray Polarimetry: A New Window in Astrophysics, eds. R. Bellazzini, et al. (Cambridge University Press), 1

Weisskopf, M. C., Silver, E. H., Kestenbaum, H. L., Long, K. S., \& Novick, R. 1978, ApJ, 220, L117

Weisskopf, M. C., Elsner, R. F., \& O’Dell, S. L. 2010, SPIE Conf. Ser., 7732 77320

Yonetoku, D., Murakami, T., Gunji, S., et al. 2013, PASJ, 63, 625

Zdziarski, A. A., Pjanka, P., Sikora, M., \& Stawarz, Ł. 2014, MNRAS, 442 3243 